\title{
ASO Author Reflections: Germline Testing for All Patients With Breast Cancer: Has the Time Finally Come?
}

\author{
Abhenil Mittal, MD, DM¹, and Raja Pramanik, MD, DM² (1) \\ ${ }^{1}$ Department of Medical Oncology and Hematology, Princess Margaret Cancer Center, University Health Network, \\ Toronto, ON, Canada; ${ }^{2}$ Department of Medical Oncology, Dr. B.R.A. Institute Rotary Cancer Hospital, All India Institute \\ of Medical Sciences, New Delhi, India
}

\begin{abstract}
For the young breast cancer population in India, the burden of hereditary breast cancer is not well defined. Moreover, genetic testing criteria (National Comprehensive Cancer Network and Mainstreaming Cancer Genetics (MCG) plus) have never been validated for the Indian population. Therefore, this study tested 236 consecutive breast cancer patients for germline pathogenic mutations using next-generation sequencing and reflex Multiplex Ligation Probe Amplification (MLPA). The findings showed a high prevalence of pathogenic/likely pathogenic (P/LP) mutations (18.64\%), with 34\% mutations in non BRCA genes. The sensitivity of the testing criteria was inadequate $(88.6 \%$ for $\mathrm{NCCN}$ and $86.36 \%$ for MCG plus criteria), reiterating the need to expand the criteria. The uptake of cascade testing was low (10\% of eligible previvors), highlighting this as an area of unmet need. Multicentric studies to validate these data and provide further insight into the hereditary cancer burden in India are the need of the hour.
\end{abstract}

\section{PAST}

India harbors a young breast cancer population versus the West, with a high prevalence of triple-negative breast cancer. ${ }^{1}$ Therefore, it is expected that the burden of hereditary breast cancer would be high in India. However,

(C) Society of Surgical Oncology 2021

First Received: 21 September 2021

Accepted: 21 September 2021;

Published Online: 15 October 2021

R. Pramanik, MD, DM

e-mail: drrajapramanik@gmail.com due to a lack of well-conducted population- and hospitalbased studies, this burden remains largely undefined. Moreover, with the decline in cost and an easier access to germline genetic testing, the question remains as to which patients would benefit the most from testing. The NCCN criteria are almost universally followed, and the practice at the majority of Indian centers is based on these criteria. However, the criteria have never been formally validated in the Indian setting. Recent Western studies have highlighted the deficiencies of the NCCN criteria when applied to a white Hispanic population and have stressed the need to test all women with breast cancer to better define risk and inform management for the patient as well as her family. ${ }^{2,3}$ This study aimed to assess the prevalence of hereditary breast cancer among Indian patients by testing all new consecutive patients and to determine the applicability of the NCCN and Mainstreaming Cancer Genetics (MCG) plus criteria for the Indian population.

\section{PRESENT}

This study recruited 236 women with breast cancer during a 1-year period. Unsurprisingly, most of the women were young (median age, 47 years; $62.9 \%<50$ years of age), and $35 \%$ had triple-negative disease. Multigene panel testing by next-generation sequencing followed by reflex Multiplex Ligation Probe Amplification (MLPA) was performed for all the patients. The findings showed an alarmingly high rate of pathogenic/likely pathogenic $(\mathrm{P} /$ LP) mutations in this cohort, with 44 (18.64\%) of 236 women having a mutation, compared with an average of $5 \%$ in the West.

Although BRCA1 and BRCA2 were the two most commonly implicated genes, $34 \%$ of the mutations were observed in non-BRCA genes, highlighting the significance 
of multigene panel testing. Testing only by NCCN criteria or MCG plus criteria would have missed five and six patients respectively (sensitivity of $88.6 \%$ and $86.36 \%$, respectively). Expanding the criteria to include all patients up to the age of 60 years could potentially increase sensitivity to $97.7 \%$. Practically, by testing 22 additional women, five more mutations could have been detected. Additionally, 10 patients underwent risk-reducing mastectomy, and 7 patients underwent risk-reducing bilateral salpingoophorectomy. Delays related to COVID-19 led to the postponement of many surgeries, which hopefully will be performed in the future.

The study also was able to perform cascade-testing for 16 families and could identify 23 unaffected previvors with mutations. However, acceptance of cascade testing was low (10\% of all eligible previvors underwent testing). The results suggest that criteria-based testing is inadequate even in the Indian context and needs expanding. ${ }^{4}$ Although testing of more women is likely to increase variant of uncertain significance (VUS) and may put further strain on health care, it needs to be integrated into routine oncologic care for all breast cancer patients. ${ }^{5}$ Enough evidence exists to suggest the cost effectiveness of this approach, and with the evidence for poly ADP ribose polymerase (PARP) inhibitors in the adjuvant setting, mutation testing results have a definite potential to impact patient management from the outset. ${ }^{6}$

\section{FUTURE}

This study recruited patients at a tertiary care referral center in North India. Although we found a high prevalence of P/LP mutations in breast cancer patients, a multicentric collaborative study including patients from all parts of India to define the hereditary cancer burden among Indian breast cancer patients and influence policy decisions is the need of the hour. Validation of these data in a larger cohort will provide confidence for clinicians to discuss genetic testing with patients early and integrate it into their treatment plan. More data on the prevalence of P/LP mutations in patients from India older than 65 years are needed to define a testing strategy for this population. Unaffected previvors need special attention, and pretest counseling for family members of affected patients needs to be prioritized.

ACKNOWLEDGMENT The funding for the study was provided by Tata Trusts at the University of Chicago, New Delhi Center.

DISCLOSURE The authors declare that they have no conflict of interest.

\section{REFERENCES}

1. Sandhu GS, Erqou S, Patterson H, Mathew A. Prevalence of triplenegative breast cancer in India: systematic review and metaanalysis. J Glob Oncol. 2016;2:412-21.

2. Beitsch PD, Whitworth PW, Hughes K, Patel R, Rosen B, Compagnoni $\mathrm{G}$, et al. Underdiagnosis of hereditary breast cancer: Are genetic testing guidelines a tool or an obstacle? J Clin Oncol. 2019;37:453-60.

3. Yang S, Axilbund JE, O'Leary E, Michalski ST, Evans R, Lincoln SE, et al. Underdiagnosis of hereditary breast and ovarian cancer in Medicare patients: genetic testing criteria miss the mark. Ann Surg Oncol. 2018;25:2925-31.

4. Mittal A, Deo SVS, Gogia A, Batra A, Kumar A, Bhoriwal S et al. Profile of pathogenic mutations and evaluation of germline genetic testing criteria in consecutive breast cancer patients treated at a North Indian tertiary care center. Ann Surg Oncol. 2021. https://d oi.org/10.1245/s10434-021-10870-w.

5. Kemp Z, Turnbull A, Yost S, Seal S, Mahamdallie S, PoyastroPearson E, et al. Evaluation of cancer-based criteria for use in mainstream BRCA1 and BRCA2 genetic testing in patients with breast cancer. JAMA Netw Open. 2019;2:e194428.

6. Tutt ANJ, Garber JE, Kaufman B, Viale G, Fumagalli D, Rastogi $\mathrm{P}$, et al. Adjuvant olaparib for patients with BRCA1- or BRCA2mutated breast cancer. OlympiA Clinical Trial Steering Committee and Investigators. N Engl J Med. 2021;384:2394-405.

Publisher's Note Springer Nature remains neutral with regard to jurisdictional claims in published maps and institutional affiliations. 